\title{
SUPERSYMMETRIC EVENT GENERATION AND THE REACH OF COLLIDERS FOR SUPERSYMMETRY
}

\author{
Howard Baer \\ Dept. of Physics, Florida State University, Tallahassee, \\ FL 92306 USA \\ Steve Mrenna \\ Argonne National Laboratory, 9700 South Cass Avenue, Argonne, \\ IL 60439 USA
}

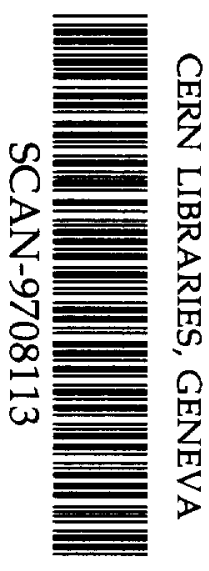

$\sin 9735$

\begin{abstract}
Models of particle physics that include weak scale supersymmetry predict a rich spectro of new particle states lying at or below the TeV energy scale. These new generator programs accessible to present or future collider experiments. Event symmetry to collider search experial tool for linking models of weak scale superstatus of event generator prexperiments. In this chapter we discuss the current ISAJET and PYTHIA programs. In additionsmmetry, focussing mainly on the collider searches at $e^{+} e^{-}$grams. In addition, we show how these can be used for popular models the regions of para colliders. As examples, we show for several discoverable above Standard Marameter space where SUSY signals ought to be NLC colliders.
\end{abstract}

\section{Introduction}

Perhaps the most compelling models of particle physics today are those that incorporate some form of weak scale supersymmetry (SUSY). ${ }^{1}$ Virtually all grand unification who make use of superstring theory, supergravity theory or of supersymmetric particles with take for granted the existence of a spectrum hand, many of their experimental colleagues $M_{\text {weak }} \sim 250 \mathrm{GeV}$. On the other cal about weak scale supersymmetry colleagues wisely choose to remain skeptiConclusive evidence will most likely until definitive evidence can be found. where superpartnes, should they come from colliding beam experiments, their properties can be measured.

Theories of weak scale supe and interactions, can seem quiterymetry, with a plethora of new particles ment of pions, electrons, muons, separated from the experimental measurelink between them is made by computer pollider detector facilities. The crucial Our task in this chapter is computer programs known as event generators. of event generator programs for d. First, we will survey the present status

This work supported in part by the U.S. Department of Energy, Division of High Energy Physics, Contract W-31-109-ENG-38. 
their utility in assessing the ability of various collider facilities to discover supersymmetry by measuring event rates of distinctive supersymmetry signatures at levels beyond those expected from background processes predicted by the Standard Model (SM).

To make predictions for sparticle production and decay rates, a specific particle physics model of supersymmetry is needed. The most conservative approach is to adopt the so-called Minimal Supersymmetric Standard Model, or MSSM. ${ }^{2}$ In this model, one assumes a superpartner for each particle of the $\mathrm{SM}$, with the same quantum numbers under the gauge symmetries $S U(3)_{C} \times$ $S U(2)_{L} \times U(1)_{Y}$. By necessity, the minimal Higgs sector contains two Higgs doublets, which leads to two neutral physical scalar bosons ( $h$ and $H$, with $m_{h}<m_{H}$ ), a neutral pseudoscalar $A$, and a pair of charged Higgs bosons $H^{ \pm}$. Because of the absence of low lying superpartner states degenerate in mass with SM particles, it is clear that SUSY must be broken. The effects of SUSY breaking are encompassed in the theory by adding all allowable soft supersymmetry breaking terms to the MSSM Lagrangian. Without further assumptions, this leads to a model with over 100 free parameters. While there are many parameters, there is no fine tuning necessary to stabilize the Higgs boson mass, which is by far the most attractive feature of weak scale SUSY. However, given such a model, how should we proceed in exploring its ramifications?

One approach is to treat the MSSM Lagrangian as an effective Lagrangian and let various experimental measurements constrain the parameters. Unless there are some relations between them and some limits on their sizes, many things will go awry. For example, some of the terms in the Lagrangian allow flavor changing neutral currents at a large rate, which must be suppressed. Others allow rapid proton decay, which has not been observed. If the MSSM is to have something to do with the weak scale, then none of these mass parameters can become too large. Such considerations can significantly reduce the number of "free" parameters. Also, some testable features of a particular model depend on only a few parameters. The D0 search for top squark pair production and decay at the Tevatron depends on only the lightest top squark mass, the lightest neutralino mass, and the assumption that all other superpartners are relatively heavy. On the other hand, an effective Lagrangian is not very satisfying, and certainly cries out for a deeper understanding. If some of the parameters are constrained to be small or vanishing, we want to understand the reason.

An important implication of minimality is that possible baryon or lepton number violating terms that can occur in the superpotential are set to zero by hand. This leads to a conserved multiplicative $R$ parity, which implies 
that sparticles must be pair produced at colliders, and that sparticles must decay to other sparticles. Thus, the lightest SUSY particle (LSP) would be absolutely stable. Arguments from cosmology and searches for exotic nuclei and atoms then imply that the LSP should be charge and color neutral. LSP's produced in sparticle cascade decays will then escape detection at collider experiments, resulting in more missing energy/momentum than that expected from neutrino production and detector resolution. Excess missing energy is the classic signature for SUSY particle production at collider experiments.

It is difficult to obtain generic predictions from the MSSM due to the large parameter space of the model. At this stage, one generally appeals to higher principles or symmetries to reduce the number of parameters needed to uniquely specify a model. A popular model is based on the ideas of supergravity and grand unification. The minimal supergravity model (mSUGRA) is a possible low energy $\left(E \leq 10^{16} \mathrm{GeV}\right.$ ) effective theory that would arise in models where gravity acts as the messenger to communicate supersymmetry breaking between the hidden and observable sectors of the model. The gravitino (the superpartner of the graviton) has a weak scale mass, but an extremely weak coupling to matter. The mSUGRA model assumes the particle content of the MSSM, and a desert between the weak and GUT scales. The assumption is then made that all scalar soft breaking masses are equal to $m_{0}$ and all gaugino soft masses equal $m_{1 / 2}$ at $M_{G U T}$. In addition, trilinear $A$ terms unify to $A_{0}$. The weak scale sparticle masses are computed by solving the renormalization group equations linking $M_{G U T}$ to $\boldsymbol{M}_{w e a k}$. Electroweak symmetry is broken radiatively by the large top quark Yukawa coupling. The parameter space of the model reduces essentially to four continuous plus one discrete parameter

$$
m_{0}, m_{1 / 2}, A_{0}, \tan \beta \text { and } \operatorname{sgn}(\mu) .
$$

Here, $\tan \beta$ is the ratio of the vacuum expectation values (vev's) of the two Higgs fields at the weak scale, and $\mu$ is the superpotential Higgsino mass term. The magnitude of $\mu$ is determined by minimization of the Higgs sector effective potential.

A recently popular alternative is given by models with gauge mediated communication of supersymmetry breaking. ${ }^{5}$ In these models, supersymmetry is again broken in a hidden sector, but now the breaking is communicated by gauge interactions. To facilitate this mechanism, additional matter multiplets are hypothesized which couple to visible matter through the SM gauge group, but also to the hidden sector. The model parameters for the minimal gaugemediated (MGM) model are taken to be

$$
\Lambda, M, \tan \beta \text { and } \operatorname{sgn}(\mu),
$$


(assuming only one set of messenger fields). In the above, $M$ is the effective scale of SUSY breaking, taken to be $M \geq 100 \mathrm{TeV}$, and $\Lambda$ is a ratio of singlet field vev's which sets the scale for the SUSY particle masses at scale $Q=M$. Electroweak symmetry is again broken radiatively. In this model, the gravitino $(\tilde{G})$ can have a mass $m_{\tilde{G}} \sim 1 \mathrm{eV}-1 \mathrm{keV}$, so that $\tilde{G}$ is the LSP.

Numerous other models are possible. Many of these reduce to the MSSM at the weak scale, but have different boundary conditions, scale choices or intermediate scale matter or gauge fields. Other models can have additional matter or gauge fields appearing at the weak scale, so that additional production and decay mechanisms beyond those of the MSSM are needed. A survey of several alternatives can be found in this volume or in the references. $6,7,8$

Currently, most event generators are set up to accommodate the physics of the MSSM, with extra switches for favorable models, such as mSUGRA and MGM. We turn next to a brief discussion of event generator programs in general, and then to specific details of the event generators ISAJET and PYTHIA.

\section{General facets of event generation}

There exist two approaches to event generation, which we characterize as exact tree-level calculations (ETC) and approximate factorized calculations (AFC). To calculate sparticle production and decay using ETC methods, one starts with a specific hard scattering production process (usually $2 \rightarrow 2$ ), as well as specific decay modes for the particles or sparticles. One may then proceed to write down the complete production and decay amplitude, including any spin correlations between initial and final state particles for the complete $2 \rightarrow n$ particle process. The matrix element can in principle be explicitly squared. However, there can exist many Feynman diagrams for production as well as decay, and in general the decays proceed through a cascade, for which there may again exist spin correlations. For such complicated configurations, it is usually more straightforward to proceed with direct numerical evaluation of helicity amplitudes. This approach has been adopted for chargino/neutralino production at the Tevatron, ${ }^{9}$ and more recently for chargino production for $e^{+} e^{-}$colliders. ${ }^{10,11}$ The advantage is that one has a complete tree level expression for production and decay. Such calculations will be necessary for precision measurements of, for instance, final state particle angular distributions once SUSY is discovered. The disadvantage is that a new calculation is needed for each specific correlated production and decay mechanism. Hence, this has been the technique of choice for studies of precision SUSY particle measurements at $e^{+} e^{-}$machines, where one is focussed on a single production mechanism 
and a simple decay mode. Such ETC calculations can be interfaced with parton shower and hadronization routines for use in detector simulations to make them more realistic.

The AFC based programs, such as ISAJET, ${ }^{12}$ PYTHIA ${ }^{13}$ and HERWIG ${ }^{14}$ (to list only the most popular) are based upon building up successive independent production and decay mechanisms. Thus, in AFC based programs, numerous different production mechanisms can be included. Particle decays are assumed to factorize into strings of 2 or 3 body decays. In this way, many strings can be patched together so that a multitude of different decay chains can be generated probabilistically. Of course, since spin correlations are neglected, the sparticle decay distributions won't be quite right. However, in many cases the sparticle decays are dominated by kinematics instead of dynamics, or in the case of scalar sparticles, there is no spin correlation. Exact decay matrix elements can be included, but are often neglected in favor of pure phase space weighting. For AFC based programs, initial and final state parton showers are included, as well as particle hadronization routines. AFC based programs are usually the program of choice for hadron colliders, where very many production mechanisms are competing, and parton distribution functions (PDF's) tend to wash out the effect of spin correlations. Since the initial state partons are colored, there are large QCD corrections to event shapes that are reproduced by parton showering. Also, AFC based programs are useful for $e^{+} e^{-}$colliders when $i$.) one is producing scalar sparticles, ii.) one has very many competing production mechanisms, iii.) the produced sparticles are so heavy that very many or very long decay cascades are operating, or $i v$.) the effects of spin correlations do not affect the particular observables one is interested in. The AFC based programs can be broken down into four distinct calculational steps which we now briefly review.

\subsection{Hard scattering}

The hard scattering is almost always a partonic $2 \rightarrow 2$ or $2 \rightarrow 3$ production subprocess calculated perturbatively using tree level Feynman diagrams. For a given kinematical configuration of the initial and final state partons, numerous possible cross sections can be computed. A random number can select which particular subprocess is to be generated based on the relative production probabilities. Of course, one must always convolute initial state quarks and gluons with an appropriate set of PDF's. In addition, some generators convolute initial state electrons and photons with distribution functions to describe the bulk effects of initial state photon radiation. The latter effect can be significant especially at very high energy $e^{+} e^{-}$colliders. For $e^{ \pm} p$ colliders, 
the hadronic structure of the photon can be included.

\subsection{Parton showers}

To properly describe jet broadening from the produced quarks and gluons, approximate all orders QCD corrections must be built in. In event generators, parton shower (PS) algorithms are used. In this case, one describes radiative quark or gluon emission using matrix elements in the collinear approximation, but with exact kinematics. These approximate matrix elements include the soft and collinear pole terms, which are the most important part of the dynamics. The divergences are regulated by cutoffs which physically correspond to lack of resolvable emissions. Thus, the parton shower can be expected to give a reasonable description of multiple parton emission. The squared matrix elements for multiple parton emissions actually factorize in the collinear limit, so that parton emission can be programmed probabilistically. For final state showers, ISAJET uses the original algorithm of Fox and Wolfram, ${ }^{15}$ while JETSET/PYTHIA and HERWIG use different algorithms incorporating angle ordering, which accounts for some extra subleading dynamics due to interference terms in multiple parton emissions. The showering schemes can also differ in their choice of evolution variables, which can depend on the virtuality of the showering parton or the energy fraction carried away by an emitted parton. Finally, all of the showering algorithms rely on an arbitrary energy cutoff that fixes the minimum energy of a showering parton.

In addition, initial state parton showers must be included. The backward shower method of Sjöstrand ${ }^{16}$ is used in ISAJET and PYTHIA, where the $Q^{2}$ dependent PDF's are actually used to compute the quark and gluon emission probabilities.

So far, no specification has been made as to the type of emitted parton. The emission of photons can be included, as well as that of gluons and quarks. In fact, it is easier to deal with photon emission, since they are produced on shell and the gauge theory is Abelian. In practice, however, initial state showering of photons is only included for lepton colliders.

\subsection{Hadronization schemes}

The process of hadronization- turning quarks and gluons into observable hadronsis not perturbatively calculable. Instead, hadronization algorithms can be constructed using various QCD inspired ideas, and by fitting functions to data. The simplest approach fragments a parton into collinear hadrons carrying a fraction of the initial parton energy or momentum. The probability of obtaining a given hadron with a given momentum fraction is determined by a 
phenomenological function called a fragmentation function, with coefficients that must be fit to data. Transverse momentum can also be added based on another function also fit to data. In the Field-Feynman (FF) independent hadronization model used in ISAJET, each parton hadronizes independently of the others. While FF generates the bulk features of hadronization, it fails to maintain color correlations and conserve energy-momentum, and the particle multiplicity is not Lorentz invariant. These problems are remedied by string fragmentation used in JETSET/PYTHIA, where partons are assumed to be linked to other partons via color strings. The string hadronization model suffers however from being non-local. The cluster hadronization model of HERWIG improves upon this by converting partons into hadrons locally in phase space.

\subsection{Beam remnants}

For hadron colliders, the beam remnants of partons not engaged in the hard scattering must be treated. This facet is again non-perturbative, so that one generally appeals to models fit to data. In ISAJET, the remnants scatter via a Pomeron exchange model. After scattering, the remnants hadronize according to a modified independent fragmentation model fit to data. In PYTHIA, a multiple scattering algorithm is used, where the multiple scatterings are adjusted again to fit data. Finally, in HERWIG, a beam remnant model based on data from experiment UA6 is used.

\subsection{Special problems for susy}

One special problem is how to define color flow in programs that keep track of color, such as PYTHIA and HERWIG. In principle, different production or decay diagrams have different color flow, and can be distinguishable, so that quantum interference terms are not used. This means that the scattering cross sections in PYTHIA may be different from those in ISAJET.

Since PYTHIA uses the color flow for string fragmentation, it needs to know the cross section for a specific color configuration. Using a $1 / N_{c}$ approximation, where $N_{c}$ is the number of colors ( 3 in this case), the exact matrix element can be projected into the different color combinations. However, there are some terms left over of order $1 / N_{c}^{2}$ which must be treated. These extra terms are redistributed according to their pole structure.

\section{Overview of ISAJET}

The program ISAJET, created by Frank Paige and Serban Protopopescu, ${ }_{1}^{12}$ was one of the first multi-purpose event generator programs to appear. It 
was originally constructed to give an idea of what jet events would look like at the ISABELLE $p p$ collider. H. Baer and X. Tata are co-authors of the supersymmetry code in ISAJET, for versions 7.00 and greater.

\subsection{Availability}

Currently, ISAJET version 7.28 is available. ISAJET is encoded using Fortran77, but is managed using the PATCHY code management system developed at CERN. The file ISAJET.CAR containing source code and PATCHY commands is available by anonymous ftp at

ftp://penguin.phy.bnl.gov:/pub/isajet. It can also be obtained via the HEP Decnet from

bnlajc: :user03: [isajet.isalibrary]. There is also a Unix makefile and a VMS is amake.com in this directory; these require that PATCEY be installed on one's system. Upon creating ISAJET, a number of files are created, including isajet.for (the main ISAJET code), ISASUSY (which generates a decay table with MSSM inputs), ISASUGRA (which solves for the particle spectra in the mSUGRA model, and lists sparticle decay modes) and ISAJET.DOC (where detailed documentation and sample programs are stored).

\subsection{The ISAJET SUSY models and sparticle decays}

Upon running the program ISASUSY, the user is prompted for an output file name, and then various sets of input parameters from the MSSM:

$$
\begin{array}{cc}
M S S M A: & m_{\dot{g}}, \mu, m_{A}, \tan \beta \\
M S S M B: & m_{Q_{1}}, m_{d_{R}}, m_{u_{R}}, m_{L_{1}}, m_{e_{R}} \\
M S S M C: & m_{Q_{1}}, m_{b_{R}}, m_{t_{R}}, m_{L_{3}}, m_{\tau_{R}}, A_{t}, A_{b}, A_{\tau} .
\end{array}
$$

In the above, $m_{\dot{g}}$ is the physical gluino mass. It undergoes a pole mass correction to derive the $S U(3)$ soft breaking gaugino mass $M_{3}$, which is then used to calculate the $S U(2)$ and $U(1)$ soft-breaking gaugino masses $M_{2}$ and $M_{1}$ via the grand unification relation. The terms in MSSMB and MSSMC above are 1st and 3rd generation soft SUSY breaking parameters: the physical masses are derived by including electroweak $D$-terms and mixings (mixings for just the 3rd generation masses). Second generation soft masses are taken by default to be equal to 1 st generation soft masses.

Optionally, one may enter the parameters

$$
M S S M D: m_{Q_{2}}, m_{t_{R}}, m_{c_{R}}, m_{L_{2}}, m_{\mu_{R}}
$$


if the model of interest has for some reason different 1 st and 2nd generation soft scalar masses. Optionally, one may also enter

$$
\text { MSSME : } M_{1}, M_{2}
$$

if one wants independent $U(1)$ and $S U(2)$ gaugino masses as input; here, $M_{1}$ and $M_{2}$ can be either positive or negative.

The ISASUSY program then calculates the complete sparticle and Higgs boson mass spectra, mixing angles and couplings, and dumps out the complete decay table for sparticles, Higgs bosons and the top quark, including the partial widths in $\mathrm{GeV}$ and branching fractions. The Higgs boson masses and couplings are calculated using the renormalization group improved one loop effective potential, including just $3 \mathrm{rd}$ generation contributions. These results agree with Ref. ${ }^{17}$ to within $2-3 \mathrm{GeV}$.

Alternatively, one can run the ISASUGRA program. In this case, the inputs are

$$
\text { SUGRA : } m_{0}, m_{1 / 2}, A_{0}, \tan \beta, \operatorname{sgn}(\mu) \text { and } m_{t} .
$$

In this case, ISASUGRA begins with GUT scale boundary conditions of the mSUGRA model and calculates the weak scale soft masses via iterative running of 26 renormalization group equations, ${ }^{18}$ using 2-loop RGE's for gauge couplings and 1-loop RGE's for Yukawas and soft terms. SUSY particle threshold effects are included. The scalar potential is minimized at scale $Q=\sqrt{m_{i_{L}} m_{i_{R}}}$, which yields a prediction of $m_{A}$ which is stable under scale variations even at large $\tan \beta$. The program then outputs various GUT and weak scale parameters, SUSY and Higgs boson masses, and the complete decay table.

ISAJET version 7.28 includes as well all 3rd generation Yukawa and mixing effects for stops, sbottoms and staus, and their associated decays. It also includes a complete set of Feynman diagrams for the 3-body $\tilde{g}, \tilde{\chi}_{1}^{ \pm}$and $\widetilde{\chi}_{2}^{0}$ decays, including all 3rd generation Yukawa and mixing effects. Finally, helicity information for subsequent tau lepton decays from $W$ 's, Higgs bosons and SUSY particles is kept, and used for subsequent event generation. ${ }^{19}$

Alternative models of weak-scale supersymmetry are usually easy to simulate in ISAJET provided they reduce to the MSSM at the weak scale. For models with gauge-mediated supersymmetry breaking, the user must provide a program to generate the appropriate sparticle masses, which can then be entered into ISAJET via MSSMi commands. Decays of the NLSP can be entered either via the FORCE command, or through explicit addition to the decay table in ISADECAY.DAT. A gravitino state is defined in ISAJET.

Models with $R$ parity violation can be simulated provided the $R$ violating couplings can be neglected in sparticle production and decay reactions, other 
than for the LSP. For the LSP, the $R$ violating decays can be explicitly added to ISADECAY.DAT.

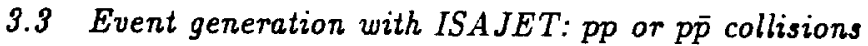

For hadron colliders, along with SM processes, ISAJET contains the following SUSY production reactions:

- $q q, g g, q g \rightarrow \tilde{g} \tilde{g}, \tilde{g} \tilde{q}, \tilde{q} \tilde{q}$, (strong production)

- $q q, q g \rightarrow \tilde{g} \bar{\chi}_{i}^{0}, \tilde{g} \tilde{\chi}_{i}^{ \pm}, \tilde{q} \tilde{\chi}_{i}^{0}, \tilde{q} \tilde{\chi}_{i}^{ \pm}$(associated production)

- $q q \rightarrow \tilde{\chi}_{i}^{ \pm} \tilde{\chi}_{j}^{\mp}, \tilde{\chi}_{i}^{ \pm} \tilde{\chi}_{j}^{0}, \tilde{\chi}_{i}^{0} \bar{\chi}_{j}^{0}$ ( $\tilde{\chi}$ pair production)

- $q q \rightarrow \tilde{\ell} \tilde{\nu}, \tilde{\ell} \tilde{\ell}, \tilde{\nu} \tilde{\nu}$ (slepton pair production).

Specific reactions can be generated via use of the ISAJET JETTYPEi command; if JETTYPE $i=$ 'ALL', then all the above subprocesses are generated according to their relative probabilities. Once produced, the sparticles decay to other sparticles via the pre-calculated branching fractions until the LSP state is reached. At this stage in ISAJET, sparticle decay distributions are generated according to phase space (although exact matrix elements are included for various SM particles such as heavy quarks and tau leptons).

In addition, the Higgs bosons of the MSSM can be produced via the direct s-channel subprocess,

- $q q, g g \rightarrow h, H, A, H^{ \pm}$

The Higgs bosons can then decay to SM or SUSY particle modes.

Finally, $t \bar{t}$ production can also be generated, including SUSY decay modes of the top quark.

\subsection{Event generation with ISAJET: $e^{+} e^{-}$collisions}

At $e^{+} e^{-}$colliders, the following production mechanisms are included:

- $e^{+} e^{-} \rightarrow \tilde{q} \overline{\tilde{q}}$ (squark pair production)

- $e^{+} e^{-} \rightarrow \overline{\tilde{\ell}}$ (slepton pair production)

- $e^{+} e^{-} \rightarrow \tilde{\chi}_{i}^{ \pm} \tilde{\chi}_{j}^{\mp}, \bar{\chi}_{i}^{0} \tilde{\chi}_{j}^{0}$ ( $\tilde{\chi}$ pair production)

- $e^{+} e^{-} \rightarrow . h Z, H Z, h A, H A, H^{+} H^{-}$(Higgs production). 
After production, the sparticles and Higgs bosons decay through the usual cascades. For $e^{+} e^{-}$collisions, the beam polarization may be specified via the ISAJET keyword EPOL, which can take values from -1 to +1 , where -1 corresponds to a pure left polarized $e$ beam, and +1 corresponds to pure right polarization. Both $e^{-}$and $e^{+}$beam polarization may be specified.

\section{Overview of PYTHIA}

PYTHIA and JETSET are programs for the generation of high-energy physics events, i.e. for the description of collisions at high energies between elementary particles such as e+, e-, p and pbar in various combinations. Together they contain theory and models for a number of physics aspects, including hard and soft interactions, parton distributions, initial and final state parton showers, multiple interactions, fragmentation and decay. They are largely based on original research, but also borrow many formulae and other knowledge from the literature.

Development of JETSET, the first member of the "Lund Monte Carlo" family, was begun by members of the Lund theory group in 1978, and has continued since then, on and off. A number of people have contributed to this and other programs based on it. The most extensive of these is PYTHIA. Over the years, the two programs have more and more come to be maintained in common. In the most recent version they have therefore been merged to one, under the PYTHIA label.

\subsection{Availability}

PYTHIA version 6.1 is the new standard distribution. The three major new aspects are: (i) the supersymmetric process machinery of SPYTHIA ${ }^{20}$ has been included, thus extending the physics range of the program; (ii) PYTHIA and JETSET have been merged, to simplify maintenance; and (iii) all real variables are declared in double precision, to improve the numerical stability at large energies.

Past and present versions, documentation, examples of use, and other useful information about PYTHIA are available on the World Wide Web at the address http://thep.lu.se/tf2/staff/torbjorn/Pythia.html. PYTHIA is written in the FORTRAI 77 computing language. The code is available in text form, gzipped text, or uuencoded gzipped text. 


\subsection{Simulating Supersymmetry with PYTHIA}

PYTHIA is run in a master-slave format, where the user is the master and PYTHIA is the slave. The user writes a main program that initializes PYTHIA by calling the subroutine PYINIT, then generates individual events by calling the subroutine PYEVIT. The PYTHIA parameters are accessed through common blocks, or, alternatively, at initialization time, through a special subroutine PYGIVE which converts characters into commands (e.g., the FORTRAI statement CALL PYGIVE('IMSS(1)=1') will set the variable IMSS $(1)=1$ ).

In general, various options are set by the array IMSS, while numerical values are fixed by the array RMSS. The level of supersymmetry simulation is set by the parameter IMSS(1)=0,1,2, which means no SUSY, a general MSSM model where all parameters must be fixed, or a mSUGRA model based on only five parameters.

For a general MSSM model (IMSS $(1)=1$ ), the elements of the array RMSS have the following meaning (the array element number is in parentheses):

(1) $M_{1}$, (2) $M_{2}$, (3) $M_{\dot{g}}$, (4) $\mu$, (5) $\tan \beta$, (6) $M_{\bar{\ell}_{L}}$, (7) $M_{\bar{\ell}_{R}}$ (8) $M_{\dot{q}_{L}}$, (9) $M_{\bar{q}_{R}}$

(10) $M_{\tilde{b}_{L}}$, (11) $M_{\tilde{b}_{R}}$, (12) $M_{\tilde{t}_{R}}$, (13) $M_{\tilde{\tau}_{L}}$, (14) $M_{\tilde{\tau}_{R}}$,

(15) $A_{b}$, (16) $A_{t}$, (17) $A_{\tau}$, (19) $M_{A}$.

Note that all of these parameters have units of mass (with the exception of $\tan \beta$, which is the ratio of mass parameters). Units of $\mathrm{GeV}$ are used in the program. Different values for the IMSS array allow many other options, such as fixing relations between $M_{1}, M_{2}$ and $M_{\dot{g}}$ or determining the third generation squark properties from the lightest sbottom and stop masses and their mixing angles. ¿From these input parameters, the complete sparticle spectrum and decay modes are calculated. The Higgs boson properties are determined using effective potential methods including the effects of third generation squarks and the chargino. ${ }^{17}$ All third generation Yukawa and mixing effects for stops, sbottoms and staus, and their associated decays, are included, as well as the complete set of Feynman diagrams for the 3-body $\tilde{g}, \tilde{\chi}_{1}^{ \pm}$and $\widetilde{\chi}_{2}^{0}$ decays, including all 3rd generation Yukawa and mixing effects.

The phenomenology of mSUGRA models can also be studied using approximate analytic formulae. ${ }^{21}$ When $\operatorname{IMSS}(1)=2$, the elements of the array RMSS have the meaning:

$$
\text { (1) } m_{1 / 2},(4) \operatorname{sgn}(\mu),(5) \tan \beta,(8) m_{0}, \text { (16) } A_{0} \text {. }
$$


All the other MSSM parameters are fixed by these few parameters, and the simulation then continues as before.

Alternative models of weak scale supersymmetry are usually easy to simulate provided they reduce to the MSSM at the weak scale. For models with gauge mediated supersymmetry breaking, the user must provide the effective MSSM parameters and the gravitino mass. All additional two body decays to the gravitino LSP are automatically calculated.

Models with $R$ parity violation can be simulated provided the $R$ violating couplings can be neglected in sparticle production and decay reactions, other than for the LSP. For the LSP, the $R$ violating decays can be explicitly simulated by updating the PYTHIA decay table with the subroutine PYUPDA.

\subsection{Event generation}

The same superpartner pair production processes in ISAJET are also available for PYTHIA. Each produced superpartner is treated as a resonance, with a mass determined by a Breit-Wigner function.

The number of production processes for the different Higgs bosons is more extensive in PYTHIA, including the associated production processes $q \bar{q}, g g \rightarrow$ $Q \bar{Q} \phi$, where $Q=b, t$ and $\phi=h, H, A, q \bar{q} \rightarrow V \phi$, where $V=W, Z$, and higher order processes.

\section{Other event generators}

A widely used event generator by LEP2 groups is called SUSYGEN ${ }^{22}$ SUSYGEN includes $e^{+} e^{-} \rightarrow S U S Y$ production and decay processes of interest to LEP2 experiments. It is set up to interface with the JETSET/PYTHIA program for parton showering and hadronization. One advantage of SUSYGEN is that it includes matrix elements for sparticle decay distributions. ${ }^{22}$

\section{Applications}

As an application, event generators can be used to estimate the reach in model parameter space for various colliders, assuming some typical integrated luminosity. Below, we present results for the LEP2, Tevatron, LHC and NLC colliders. Most of the work presented is for the mSUGRA model, although some is for the MGM model. 


\section{$6.1 \quad L E P 2$}

At LEP2, the most promising reactions for supersymmetry in the mSUGRA model are i.) light Higgs boson production $e^{+} e^{-} \rightarrow Z h$, ii.) chargino pair production $\left.e^{+} e^{-} \rightarrow \tilde{\chi}_{1}^{ \pm} \tilde{\chi}_{1}^{\mp}, i i i.\right)$ slepton pair production, e.g. $e^{+} e^{-} \rightarrow \tilde{e}_{R} \tilde{e}_{R}$ and neutralino pair production iv.) $e^{+} e^{-} \rightarrow \widetilde{\chi}_{1}^{0} \widetilde{\chi}_{2}^{0}$. Backgrounds come from various SM processes, especially $W W$ and $Z Z$ production.

In Fig. 1, we show the regions of the $m_{0}$ vs. $m_{1 / 2}$ plane where various SUSY particle reactions are observable at the $5 \sigma$ level above SM and SUSY backgrounds for an integrated luminosity of $500 \mathrm{pb}^{-1}$, and for $A_{0}=0, \mu<0$ and $m_{t}=170 \mathrm{GeV}$. The energy of LEP2 is assumed to be $\sqrt{s}=190 \mathrm{GeV}$. The regions labelled by $\mathrm{TH}$ are excluded by lack of radiative electroweak breaking or a charged or colored LSP. The EX regions are excluded by SUSY searches from LEP1. We see that most of the region below $m_{1 / 2}=100 \mathrm{GeV}$ should be explorable via the single lepton plus jets channel from chargino searches; this corresponds to a reach in $m_{\tilde{\chi}_{1}^{ \pm}} \simeq 95 \mathrm{GeV}$, i.e. saturating the kinematic limit. In addition, the lower left region of the plane can be explored via the di-electron plus missing energy channel from selectrons. There exists a small intermediate region where the dilepton signal from $\bar{\chi}_{1}^{0} \widetilde{\chi}_{2}^{0}$ production is visible. Not shown is the reach for SUSY in the $Z h$ channel. In fact, the reach of LEP2 in this channel exceeds $m_{1 / 2} \simeq 350 \mathrm{GeV}$, thus filling the whole plane shown. For other $\tan \beta$ and $A_{0}$ values, the reach is similar for the chargino and selectron detection, while the $h$ mass is typically heavier, making it more difficult to detect. ${ }^{23}$ Other detailed studies for more general models can be found in Ref. ${ }^{24}$

\subsection{Tevatron}

The most favorable mSUGRA search modes for the Fermilab Tevatron $p \bar{p}$ collider are $i$.) the missing energy plus jets mode, from squark and gluino production and $i i$.) the clean trilepton search mode $\tilde{\chi}_{1}^{ \pm} \bar{\chi}_{2}^{0} \rightarrow 3 \ell+B_{T}{ }^{9,25}$ In Fig. 2 , we show the parameter space points where SUSY signals ought to be observable above SM backgrounds. ${ }^{26}$ The cross hatched region is excluded by LEP 1.5 searches. The black squares denote mSUGRA model points discoverable beyond the $5 \sigma$ level with only $0.1 \mathrm{fb}^{-1}$ of integrated luminosity (corresponding to Run I). In this case, the discovery channel is from the jets $+B_{T}$ channel. For $2 \mathrm{fb}^{-1}$, the discovery region is denoted by grey squares; in this case, most of the reach actually comes from the clean trilepton channel. The uppermost grey squares at $m_{1 / 2}=250 \mathrm{GeV}$ correspond to a gluino mass of $650 \mathrm{GeV}$ and a chargino of mass $190 \mathrm{GeV}$ - well beyond the reach of LEP2. The white squares denote points accessible to the TeV33 upgrade, with projected inte- 
grated luminosity of $25 \mathrm{fb}^{-1}$. The TeV33 has an expanded reach mainly via the clean trilepton channel. Note however that there exist points with large $m_{0}$ where the $\widetilde{\chi}_{2}^{0}$ leptonic branching fraction drops to very low values, so that the reach of TeV33 is worse than that from LEP2 at $\sqrt{3}=190 \mathrm{GeV}$ in this region.

For the MGM model, the reach of the Tevatron can be exhibited in terms of the parameter $\Lambda$, which sets the scale for all the SUSY particle masses at the effective SUSY breaking scale $M$. A lightest chargino mass of roughly 100 $\mathrm{GeV}$ in these models can be excluded in a fairly model independent manner by studying $\gamma \gamma \mathbb{F}_{T}$ events. ${ }^{27}$ In Ref. ${ }^{28}$, the reach of the Tevatron has been estimated for the minimal gauge mediated model in terms of isolated photon plus jets plus leptons plus missing energy channels. Their calculated reach corresponds to $\Lambda \sim 100$ (135) TeV for the Tevatron $\mathrm{MI}$ ( $\mathrm{TeV} 33$ ). These reaches correspond to a reach in $m_{\dot{g}}$ of $800(1000) \mathrm{GeV}$ !

\section{$6.3 \mathrm{LHC}$}

The CERN Large Hadron Collider (LHC) is a pp collider which is under construction. It is expected to turn on around the year 2005, with projected energy of $14 \mathrm{TeV}$ and initial luminosity of about $10 \mathrm{fb}^{-1}$ per year. If weakscale supersymmetry exists, a wide variety of signals are expected to be observable. For the mSUGRA model, these consist mainly of various multi-jet plus multi-lepton plus missing transverse energy events. Various signals and SM backgrounds have been calculated in the mSUGRA model using ISAJET, and the reach contours are presented in Fig. 3 in the $m_{0}$ vs. $m_{1 / 2}$ plane, for $A_{0}=0, \tan \beta=2$ and $\mu>0 .^{29}$ The region in the lower-left corner denoted by $l$ is where the dilepton plus missing energy signal from direct production of sleptons should be visible: this essentially covers the region where $m_{i}<300$ $\mathrm{GeV}$, which is the region favored by neutralino relic density calculations. ${ }^{30}$ The region denoted by $\tilde{\chi}_{1}^{ \pm} \widetilde{\chi}_{2}^{0}$ is where jet-free $\tilde{\chi}_{1}^{ \pm} \bar{\chi}_{2}^{0} \rightarrow 3 \ell$ signals ought to be visible above SM and SUSY backgrounds. Note, as for the Tevatron cases, the gap around $m_{0} \sim 500 \mathrm{GeV}$ and $m_{1 / 2}<200$ where this signal is not visible.

The highest contour labelled by $1 \ell$ is where single isolated lepton plus jets plus $B_{T}$ is visible above SM background, and gives the maximum reach of the LHC- all the way out to $m_{\dot{g}}>2 \mathrm{TeV}$ for the LHC low luminosity option. Comparing this reach to expectations from fine-tuning and relic density estimates shows that the LHC should be able to either discover or rule out weak scale supersymmetry, at least in its mSUGRA manifestation! The contour labelled $B_{T}$ is where multi-jet plus $B_{T}$ signals (no isolated leptons) should be visible. The SS, OS and $3 \ell$ contours all show the reach for mSUGRA in 
the same-sign dilepton, opposite sign dilepton and trilepton channels, where each of these are accompanied by jets and missing transverse energy. By comparing various combinations of energy and mass distributions with model expectations, various constraints on SUSY particle masses and parameters ought to be obtainable: see Ref. ${ }^{31}$ for some detailed case studies.

\section{$6.4 N L C$}

Many physicists are interested in constructing a very high energy linear $e^{+} e^{-}$ collider (the NLC, or Next Linear Collider), with possible center of mass energy options of $0.5,1$ and $1.5 \mathrm{TeV}$. The reach of such a collider for the mSUGRA model has been calculated in Ref. ${ }^{32}$ using ISAJET. For instance, assuming an NLC with $\sqrt{s}=0.5 \mathrm{TeV}$ and integrated luminosity of $20 \mathrm{fb}^{-1}$, the lightest Higgs scalar $h$ should be visible over the whole parameter space. Thus, if a light scalar Higgs is not found at the NLC, then the mSUGRA model would be excluded. A reach contour for NLC for SUSY particle discovery is shown in Fig. 4. NLC500 should see chargino pairs at $5 \sigma$ above SM background for $m_{1 / 2} \leqslant 300 \mathrm{GeV}$ and $m_{0} \gtrsim 200 \mathrm{GeV}$. In addition, selectron pairs ought to be visible in the low $m_{0}$ region up to $m_{1 / 2} \simeq 520 \mathrm{GeV}$. The bulge in the NLC500 contour around $m_{0} \sim 300 \mathrm{GeV}$ and $m_{1 / 2} \sim 350 \mathrm{GeV}$ is where $\widetilde{\chi}_{1}^{0} \widetilde{\chi}_{2}^{0} \rightarrow b \bar{b}+B \overline{\text { is }}$ visible. The reach contour is nearly independent of whether the NLC achieves the capability of beam polarization.

Approximate reach contours are plotted in Fig. 4 for NLC at $\sqrt{s}=1000$ $\mathrm{GeV}(\mathrm{NLC1000})$ and $\sqrt{s}=1500 \mathrm{GeV}(\mathrm{NLC1500})$. For these cases, detailed signal-to-background studies were not made, but instead the reach was obtained by just plotting where $m_{i}$ and $m_{\tilde{\chi}_{1}^{ \pm}}$were equal to the beam energy.

\subsection{Comparison of reach in the mSUGRA model}

In Fig. 4, we show for comparison purposes the reach of the Tevatron MI, TeV33, the LHC with $10 \mathrm{fb}^{-1}$ of integrated luminosity, and the NLC500, NLC1000 and NLC1500 options. For perspective, we also show contours where $m_{\dot{q}}$ and $m_{\dot{q}}$ equal $1000 \mathrm{GeV}$. On this expanded scale, the Tevatron options appear to only have a small reach for SUSY; however, these regions are exactly those most favored by fine tuning constraints. ${ }^{33}$ The LHC collider can explore well beyond the $1 \mathrm{TeV}$ mass scale for supersymmetry (note this statement holds even if baryon number ( $R$ violating) LSP decays occur. ${ }^{34}$ ). If $L H C$ fails to find SUSY, it will be very difficult to understand how weak scale supersymmetry can exist. We also see from Fig. 4 that the reach of LHC is similar to that of a NLC collider at $\sqrt{s} \simeq 1000-1500 \mathrm{GeV}$. The NLC collider options have 
the advantage in that if superparticles are discovered, then relatively model independent precision measurements of sparticle masses and parameters can be made. ${ }^{10,32,35}$

\section{Conclusion}

It is only within the past several years that realistic event generation of supersymmetric particles has been possible. The capability of supersymmetric event generation in ISAJET and PYTHIA is relatively new, and both programs are undergoing evolution and change to improve on the physics input and the variety of models available for use. However, the significant progress that has occured has allowed reliable calculations to be made of how supersymmetry would be revealed in colliding beam experiments.

\section{Acknowledgments}

This report was supported in part by the U. S. Department of Energy under grant number DE-FG-87ER40319. The work of SM was supported by DOE grant W-31-109-ENG-38.

\section{References}

1. See, e.g. H. Haber in Woodlands Superworld, hep-ph/9308209 (1993).

2. For a review of the minimal model and SUSY phenomenology, see $\mathrm{H}$. Haber and G. Kane, Phys. Rep. 117, 75 (1985); X. Tata, in The Standard Model and Beyond, p. 304, edited by J. E. Kim, World Scientific (1991); M. Drees, APCTP-05 (lectures given at Inauguration Conference of the Asia Pacific Center for Theoretical Physics (APCTP), Seoul, Korea (1996), hep-ph/9611409.

3. U. Amaldi, W. de Boer and H. Fürstenau, Phys. Lett. B260, 447 (1991); J. Ellis, S. Kelley and D. Nanopoulos, Phys. Lett. B260, 131 (1991); P. Langacker and M. Luo, Phys. Rev. D44, 817 (1991).

4. A. Chamseddine, R. Arnowitt and P. Nath, Phys. Rev. Lett. 49, 970 (1982); R. Barbieri, S. Ferrara and C. Savoy, Phys. Lett. B119, 343 (1982); L.J. Hall, J. Lykken and S. Weinberg, Phys. Rev. D27, 2359 (1983); for a recent review, see e.g. M. Drees and S. Martin in Electroweak Symmetry Breaking and New Physics at the TeV Scale, edited by $\mathrm{T}$. Barklow, S. Dawson, H . Haber and J. Seigrist, (World Scientific) 1995.

5. M. Dine and A. Nelson, Phys. Rev. D48, 1277 (1993); M. Dine, A. Nelson, Y. Shirman, Phys. Rev. D51, 1362 (1995); M. Dine, A. Nelson, 
Y. Nir and Y. Shirman, Phys. Rev. D53, 2658 (1996).

6. J. Amundson et al., hep-ph/9609374 (1996).

7. M. Peskin, hep-ph $/ 9604339$.

8. J. L. Hewett and T. Rizzo, Phys. Rept. 183, 193 (1989).

9. H. Baer, K. Hagiwara and X. Tata, Phys. Rev. Lett. 57, 294 (1986) and Phys. Rev. D35, 1598 (19987).

10. T. Tsukamoto, K. Fujii, H. Murayama, M. Yamaguchi and Y. Okada, Phys. Rev. D51, 3153 (1995); see also JLC-1, KEK Report 92-16 (1992).

11. J. L. Feng and D. E. Finnell, Phys. Rev. D49, 2369 (1994).

12. F. Paige and S. Protopopescu, in Supercollider Physics, p. 41, ed. D. Soper (World Scientific, 1986); H. Baer, F. Paige, S. Protopopescu and X. Tata, in Proceedings of the Workshop on Physics at Current Accelerators and Supercolliders, ed. J. Hewett, A. White and D. Zeppenfeld, (Argonne National Laboratory, 1993), hep-ph/9305342.

13. T. Sjöstrand, Computer Phys. Commun. 82, 74 (1994).

14. G. Marchesini, B. Webber, G. Abbiendi, I. Knowles, M. Seymour and L. Stanco, Computer Phys. Commun. 67, 465 (1992).

15. G. Fox and S. Wolfram, Nucl. Phys. B168, 285 (1980).

16. T. Sjöstrand, Phys. Lett. 157B, 321 (1985); T. Gottschalk, Nucl. Phys. B277, 700 (1986).

17. M. Carena, J. Espinosa, M. Quiros and C. Wagner, Phys. Lett. B355, 209 (1995); M. Carena, M. Quiros and C. Wagner, Nucl. Phys. B461, 407 (1996); H. Haber, R. Hemplling and A. Hoang, hep-ph/9609331 (1996).

18. H. Baer, C. H. Chen, R. Munroe, F. Paige and X. Tata, Phys. Rev. D51, 1046 (1995).

19. H. Baer, C. H. Chen, M. Drees, F. Paige and X. Tata, hep-ph/9704457 (1997).

20. S. Mrenna, to appear in Comput. Physics. Commun. (1997).

21. M. Drees and S.P. Martin, hep-ph/9504324 (unpublished).

22. S. Katsanevas and S. Melachroinos, SUSYGEN.

23. H. Baer, M. Brhlik, R. Munroe and X. Tata, Phys. Rev. D52, 5031 (1995).

24. G. F. Giudice et al., hep-ph/9602207 (1996)

25. See Ref. ${ }^{9}$ and also R. Arnowitt and P. Nath, Mod. Phys. Lett. A2, 331 (1987); R. Barbieri, F. Caravaglios, M. Frigeni and M. Mangano, Nucl. Phys. B367, 28 (1993); H. Baer and X. Tata, Phys. Rev. D47, 2739 (1993); J. Lopez, D. Nanopoulos, X. Wang and A. Zichichi, Phys. Rev. D48, 2062 (1993) and Phys. Rev. D52, 142 (1995); J. Lopez, D. Nanopoulos, G. Park, X. Wang and A. Zichichi, Phys. Rev. D50, 2164 
(1994); H. Baer, C. Kao and X. Tata, Phys. Rev. D48, 5175 (1993); S. Mrenna, G. Kane, G. Kribs and J. Wells, Phys. Rev. D53, 1168 (1996).

26. H. Baer, C. H. Chen, C. Kao and X. Tata, Phys. Rev. D52, 1565 (1995); H. Baer, C. H. Chen, F. Paige and X. Tata, Phys. Rev. D54, 5866 (1996).

27. S. Ambrosanio, G.L. Kane, G.D. Kribs, S.P. Martin, and S. Mrenna, Phys. Rev. D54, 5395 (1996).

28. H. Baer, M. Brhlik, C. H. Chen and X. Tata, Phys. Rev. D55, 4463 (1997).

29. H. Baer, C. H. Chen, F. Paige and X. Tata, Phys. Rev. D52, 2746 (1995) and Phys. Rev. D53, 6241 (1996).

30. H. Baer and M. Brhlik, Phys. Rev, D53, 597 (1996).

31. A. Bartl et al., LBL-39413 (1996) and I. Hinchliffe, F Paige, M. Shapiro, J. Soderqvist and W. Yao, Phys. Rev. D55, 5520 (1997).

32. H. Baer, R. Munroe and X. Tata, Phys. Rev. D54, 6735 (1996).

33. For fine-tuning constraints, see e.g. G. Anderson and D. Castaño, Phys. Rev. D52, 1693 (1995).

34. H. Baer, C. H. Chen, and X. Tata, Phys. Rev. D55, 1466 (1997).

35. M. N. Danielson et al., Supersymmetry at the NLC, proceedings of 1996 Snowmass workshop.

Figure 1: Reach of LEP2 collider for SUSY

Figure 2: Reach of Tevatron collider for SUSY

Figure 3: Reach of LHC pp collider for SUSY 
Figure 4: Reach of Tevatron Mnin Injector, TeV33, LHC and various energy options for a NLC linear $e^{+} e^{-}$collider for SUSY 


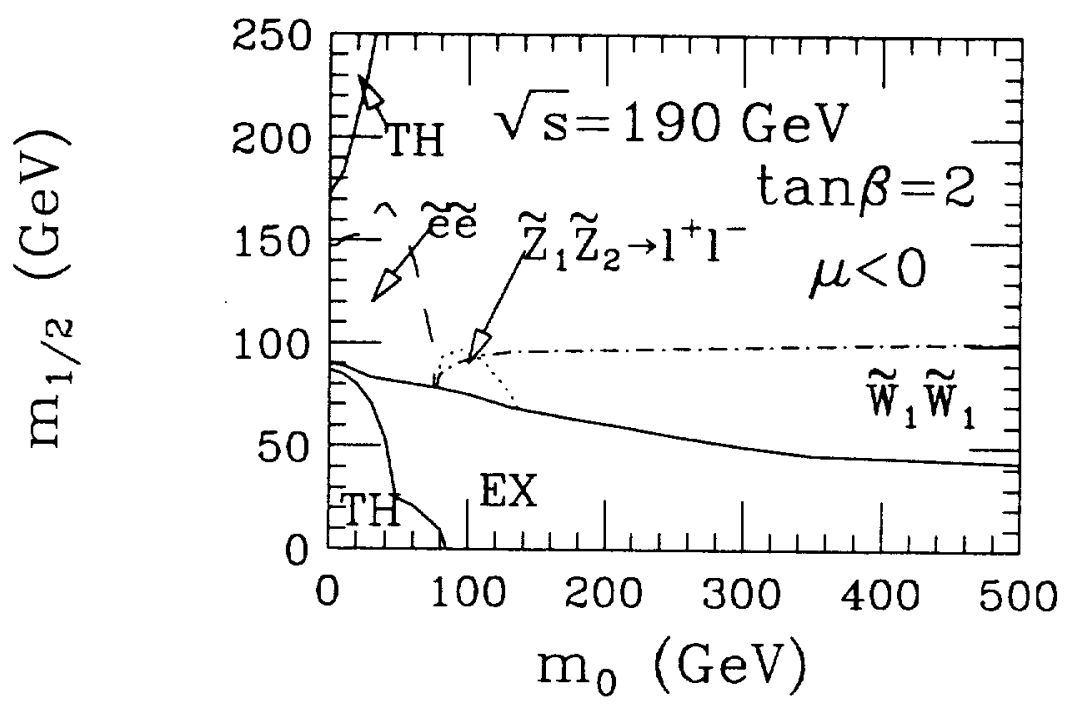




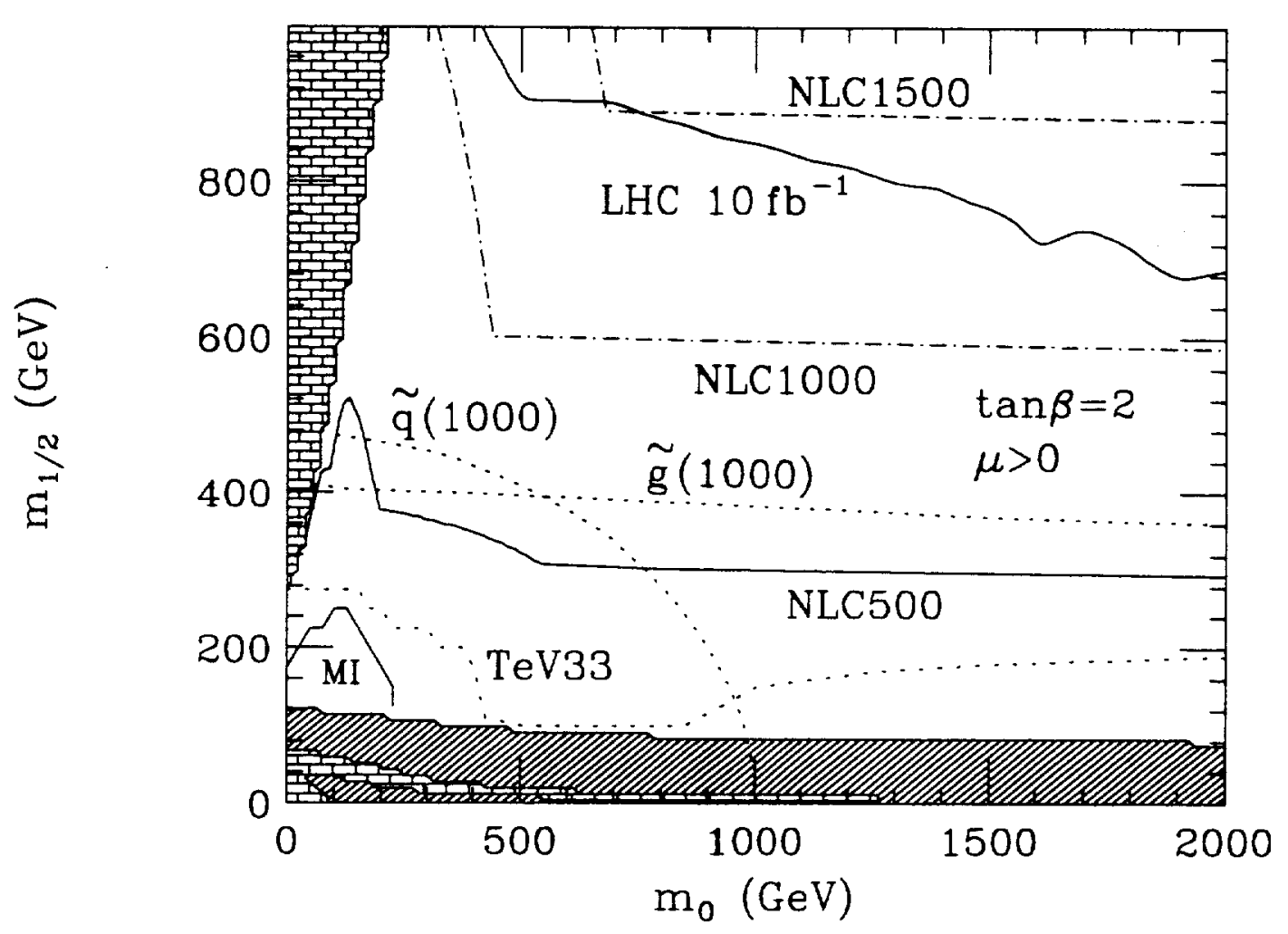




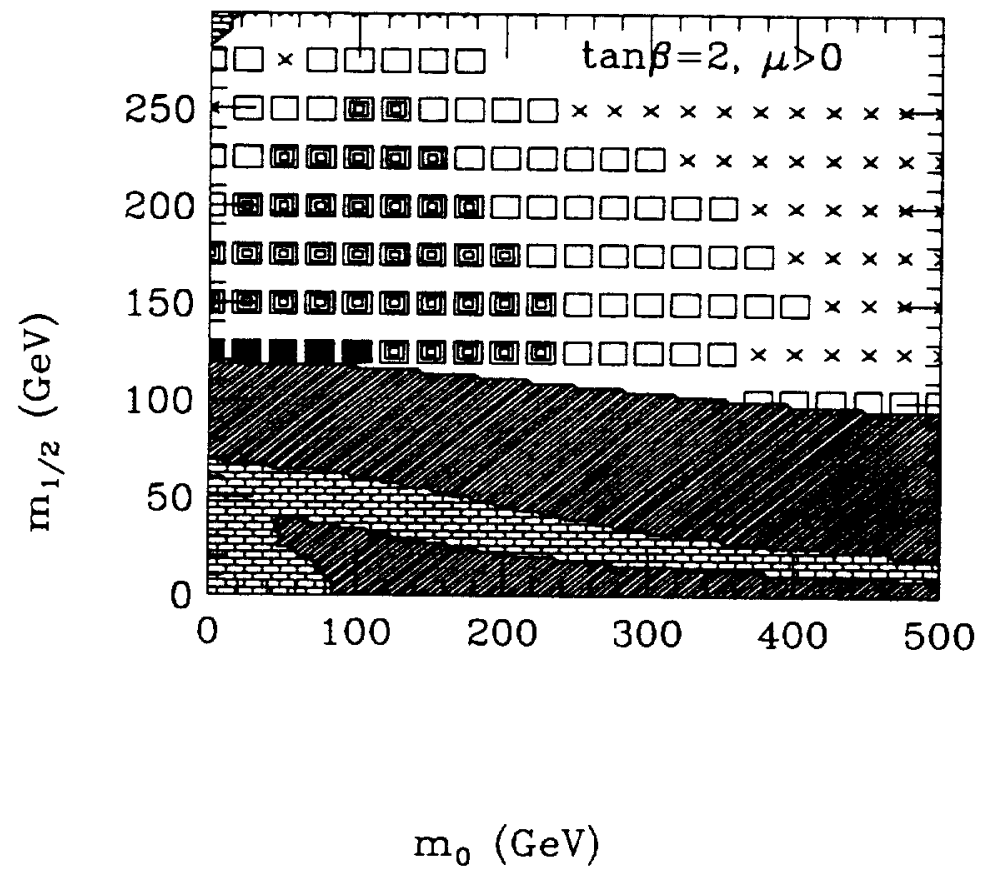




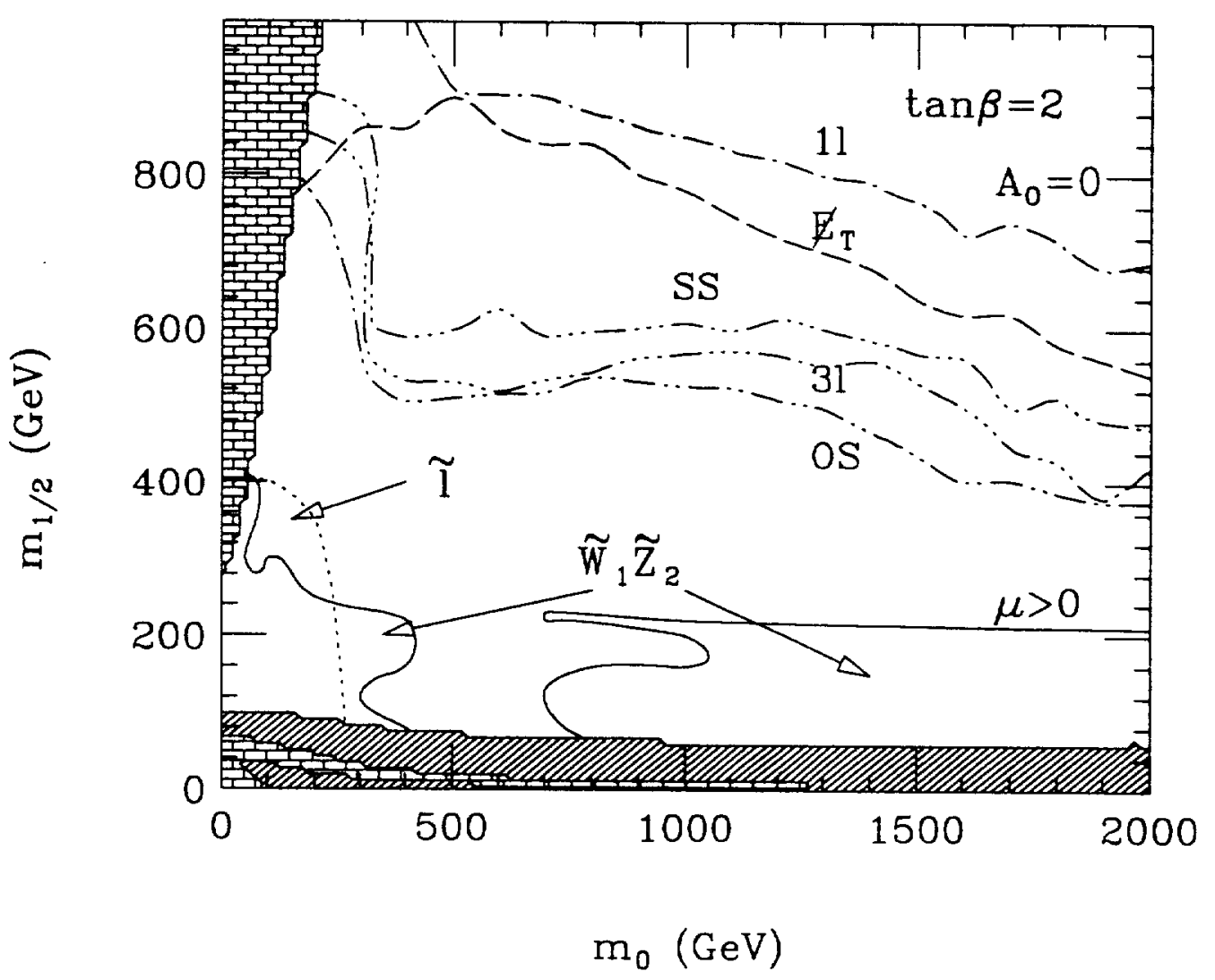

